From the Tufts Center for the Study of Drug Development, Tufts University, Boston, MA; Department of Economics, Duke University, Durham, NC.

Submitted September 5, 2006; accepted September 26, 2006

Authors' disclosures of potential conflicts of interest and author contributions are found at the end of this article.

Address reprint requests to Joseph A. DiMasi, PhD, Tufts Center for the Study of Drug Development, Tufts University, 192 South St, Suite 550, Boston, MA 02111; e-mail: joseph.dimasi@tufts.edu.

(C) 2007 by American Society of Clinical Oncology

0732-183X/07/2502-209/\$20.00

DOI: $10.1200 / J C 0.2006 .09 .0803$

\title{
Economics of New Oncology Drug Development
}

\author{
Joseph A. DiMasi and Henry G. Grabowski
}

$$
\begin{array}{llllllll}
\text { A } & \text { B } & \text { S } & \text { T } & \text { R } & \text { A } & \text { C } & \text { T }
\end{array}
$$

\section{Purpose}

Review existing studies and provide new results on the development, regulatory, and market aspects of new oncology drug development.

\section{Methods}

We utilized data from the US Food and Drug Administration (FDA), company surveys, and publicly available commercial business intelligence databases on new oncology drugs approved in the United States and on investigational oncology drugs to estimate average development and regulatory approval times, clinical approval success rates, first-in-class status, and global market diffusion.

\section{Results}

We found that approved new oncology drugs to have a disproportionately high share of FDA priority review ratings, of orphan drug designations at approval, and of drugs that were granted inclusion in at least one of the FDA's expedited access programs. US regulatory approval times were shorter, on average, for oncology drugs ( 0.5 years), but US clinical development times were longer on average (1.5 years). Clinical approval success rates were similar for oncology and other drugs, but proportionately more of the oncology failures reached expensive late-stage clinical testing before being abandoned. In relation to other drugs, new oncology drug approvals were more often first-in-class and diffused more widely across important international markets.

\section{Conclusion}

The market success of oncology drugs has induced a substantial amount of investment in oncology drug development in the last decade or so. However, given the great need for further progress, the extent to which efforts to develop new oncology drugs will grow depends on future public-sector investment in basic research, developments in translational medicine, and regulatory reforms that advance drug-development science.

\section{J Clin Oncol 25:209-216. (C) 2007 by American Society of Clinical Oncology}

\section{INTRODUCTION}

Although progress has been made in treating many forms of cancer, there remains a strong medical need for substantial improvement. This makes the complex economics of new oncology drug development an important area to research. In recent years, rising prices and growing expenditures on oncology drugs ${ }^{1}$ have caused significant concern among payers and patients. ${ }^{2}$ At the same time, and likely due in part to expanded market opportunities, some data indicate that the development of new, often targeted, oncology therapies has recently been growing significantly. ${ }^{3,4}$ The extent to which markets will grow in the future, however, is uncertain because sponsors may face increasing resistance to what are perceived to be high and unsustainable prices, increasing competition if a substantial number of new therapies enter the market, and smaller market sizes for highly targeted therapies. ${ }^{4}$
Incentives to develop new therapies also depend on the costs, risks, and length of new drug development. Pharmaceutical research and development (R\&D) costs in general have been estimated to be high and rising substantially over time. ${ }^{5}$ Costs (at least clinical phase expenditures) have also been shown to differ by therapeutic class. ${ }^{6}$ Unfortunately, to date, not enough information has been available to reliably estimate $\mathrm{R} \& \mathrm{D}$ costs for oncology drugs. A good deal of information, however, can be gathered on other metrics of the drug development process for oncology drugs. This article will review information on the markets for new oncology drugs and present new data on the length and risks of new oncology drug development.

\section{METHODS}

To analyze various aspects of the development, regulatory, and market characteristics of new oncology drugs, we 
utilized a variety of data sources. Information on new drug US clinical development and approval times were obtained from public sources and company surveys, and were complied for a Tufts Center for the Study of Drug development (CSDD) database. The US clinical phase is defined here as the time from first filing of an investigational new drug application (IND) with the US Food and Drug Administration (FDA) to study a new drug in humans to first submission to the FDA of a new drug application (NDA) or biologic license application (BLA) for marketing approval of the new drug. The approval phase is the time from first submission of an NDA or BLA to approval of the application for marketing. With regard to these development and approval times, we focus attention on therapeutic drugs and biologics that had first obtained FDA approval for US marketing from 1990 through 2005. We examined both new chemical entities and therapeutically significant new biologics. For the sake of brevity in expression, we refer to all of these compounds as new drugs. We have public NDA/BLA submission and approval dates for all of these new drugs, and dates of first IND filing (which have been validated with the FDA) for $95 \%$ of these compounds.

We analyzed clinical approval success rates based on information obtained from a publicly available business intelligence database (IMS Health's $R \& D$ Focus) for the 20 largest pharmaceutical firms in terms of pharmaceutical sales in 2005, with supplementary information from other commercial business intelligence databases. Given the lengthy development process, only compounds that had entered clinical testing through 2002 were included in the phase transition probability analyses. Their status was tracked through the first half of 2006. In addition, because a relatively large share of the compounds that initiated clinical testing during the latter half of the success rate analysis period are still active, a separate analysis for the 1998 through 2002 period would be questionable. Instead, to obtain a sense for the direction and extent of changes over time we compared results for the entire 1993 to 2002 period with results for the 1993 to 1997 period.

A number of studies of drug industry success rates have used statistical inference techniques (mainly survival analysis) to account for the rightcensoring of the data. ${ }^{5,11}$ However, given the relatively recent experience of the compounds we considered here and the length of the development process for many drugs, a significant number of compounds that we examined had not yet reached their final fate (abandonment or marketing approval), thereby making these statistical approaches somewhat unreliable. Therefore, we estimate success and phase attrition rates in a mechanistic manner. Specifically, we calculated phase transition probabilities by dividing the number of molecules that completed a given phase and entered the next phase by the difference between the number of molecules that entered the phase and those still in the phase at the time of the analysis. Such an approach should provide reasonable estimates of phase transition probabilities because the lengths of individual phases are short relative to total development times. The accuracy depends on an implicit assumption that those drugs that are still active at the time of analysis will proceed to later phases more or less in the same proportions as the estimated transition probabilities. The overall clinical success rate is then determined as the product of the phase transition probabilities. Clinical success is defined as US regulatory approval for marketing.

Data on market and other characteristics of new drug launches were obtained from IMS Health's New Product Focus database used for a study of the quality and quantity of worldwide new drug introductions. ${ }^{12}$ This database reports drug launches in 68 countries since 1982. The data examined includes new biologic products, but it excludes diagnostic tests (except for radiopaques), radiologicals, over-the-counter drugs, combination vaccines, polyclonal antibodies, and biologic extracts. Launch dates were used to determine whether a new drug launch was for a first-in-class drug. Therapeutic classes for this analysis were chosen based on a unique combination of the four-digit level Anatomic Therapeutic Classification (ATC) and five-digit level Uniform System of Classification (USC) codes. The ATC and USC system are the same for many therapeutic classes, but when they differed, as a general principle the most disaggregate class from these two sources was used.

\section{RESULTS}

We first examined the number and regulatory characteristics of new oncology drug approvals in the Unites States since 1990. Table 1 lists the 68 new oncology drugs approved for marketing in the United States from 1990 to 2005, along with their NDA/BLA submission and approval dates. The FDA also approved 434 other new drugs (as defined herein) during this period. Seventy-nine percent of the approved new oncology drugs are traditional small-molecule compounds $(78 \%$ of the other new drugs approved during the study period are also small molecules). If we narrow the focus on largemolecule approvals to the most common types of approved "biotech" products (recombinant proteins and monoclonal antibodies [mAbs]; excluding, for example, purified biologics), we find that $18 \%$ of the oncology drug approvals and 15\% of the other drug approvals are biotech products under this definition. The biotech share of all drug approvals increased over time for both oncology and other drugs, although the rate of increase was faster for oncology drugs. The biotech shares were 8\% and 9\% during 1990 to 1993 for oncology and other drugs, respectively. However, the biotech shares rose to $29 \%$ and $24 \%$ during 2002 to 2005 for oncology and other drugs, respectively.

From a regulatory perspective, the oncology drugs differ markedly from other new drug approvals. As Table 2 indicates, $71 \%$ of the oncology drug approvals were given a priority review rating by the FDA, in contrast to $40 \%$ for other new drugs. Nearly half of the oncology drugs were initially approved with an orphan drug indication, while less than one in five other drugs had orphan drug status at first approval. Finally, sponsors of oncology drugs were much more often able to take advantage of at least one of the FDA's programs to speed development (subpart E, accelerated approval, fast track). Close to half of the approved oncology drugs had some expedited access status during development, as opposed to only $13 \%$ for the other new drugs approved during the study period.

\section{Oncology Drug Development Times}

As noted, oncology drugs are disproportionately given priority ratings by the FDA, which carries with it a performance goal for faster review of marketing applications. This is reflected in the approval phase means shown in Figure 1. The FDA reviewed oncology drugs, on average, 6 months faster than other drugs. We also noted that oncology drugs were more likely to be able to take advantage of FDA expedited access programs during development. However, despite this fact, difficulties in recruiting patients and longer times needed to establish efficacy (particularly if survival is an end point) for oncology drug clinical trials can help explain why we found US clinical development times to be a year and a half longer for oncology drugs. For the period analyzed, oncology drugs took, on average, 1 year longer to move from the initiation of clinical testing in the United States to US regulatory marketing approval. Development and approval phase times are lower for medians, but the comparative results are similar. Median approval phase times are 0.3 years shorter for oncology drugs (1.0 $v 1.3$ years), whereas median clinical phase times are 1.5 years longer for oncology drugs (7.8 $v 6.3$ years).

\section{Technical Success Rates for Oncology Drug Development}

To examine technical success rates and phase transition rates for investigational oncology and other drugs, we obtained data on the pipelines of the 20 pharmaceutical firms with the most 
Table 1. New Oncology Compounds Approved in the United States, 1990-2005

\begin{tabular}{|c|c|c|c|c|}
\hline Generic Name & Trade Name & Sponsor & $\begin{array}{l}\text { NDA Submission } \\
\text { Date }\end{array}$ & $\begin{array}{c}\text { NDA Approval } \\
\text { Date }\end{array}$ \\
\hline Abarelix & Plenaxis & Praecis & $12 / 12 / 2000$ & $11 / 25 / 2003$ \\
\hline Aldesleukin & Proleukin & Chiron & 12/1/1988 & $5 / 5 / 1992$ \\
\hline Alemtuzumab & Campath & Berlex & 12/23/1999 & $5 / 7 / 2001$ \\
\hline Alfuzosin & Uroxatral & Sanofi-Synthelabo & $12 / 8 / 2000$ & $6 / 12 / 2003$ \\
\hline Alitretinoin & Panretin & Ligand & $5 / 27 / 1998$ & 2/2/1999 \\
\hline Altretamine & Hexalen & U.S. Bioscience & 12/19/1988 & 12/26/1990 \\
\hline Amifostine & Ethyol & U.S. Bioscience & 9/30/1991 & 12/8/1995 \\
\hline Aminolevulinic acid & Levulan Kerastick & Dusa & 7/1/1998 & 12/3/1999 \\
\hline Anastrozole & Arimidex & Zeneca & 3/29/1995 & 12/27/1995 \\
\hline Aprepitant & Emend & Merck & $9 / 27 / 2002$ & $3 / 26 / 2003$ \\
\hline Arsenic trioxide & Trisenox & Cell Therapeutics & $3 / 28 / 2000$ & $9 / 25 / 2000$ \\
\hline Azacitidine & Vidaza & Pharmion & $12 / 29 / 2003$ & $5 / 19 / 2004$ \\
\hline Bcg, live & Pacis & Biochem Pharma & 4/21/1995 & $3 / 9 / 2000$ \\
\hline Bevacizumab & Avastin & Genentech & $9 / 30 / 2003$ & $2 / 26 / 2004$ \\
\hline Bexarotene & Targretin & Ligand & 6/23/1999 & 12/29/1999 \\
\hline Bicalutamide & Casodex & Zeneca & 9/14/1994 & 10/4/1995 \\
\hline Bortezomib & Velcade & Millennium & $1 / 21 / 2003$ & $5 / 13 / 2003$ \\
\hline Capecitabine & Xeloda & Roche & 10/31/1997 & 4/30/1998 \\
\hline Cetuximab & Erbitux & Imclone & $8 / 14 / 2003$ & $2 / 12 / 2004$ \\
\hline Cladribine & Leustatin & Ortho & 12/31/1991 & 2/26/1993 \\
\hline Clofarabine & Clolar & Genzyme & $3 / 30 / 2004$ & $12 / 28 / 2004$ \\
\hline Denileukin diftotox & Ontak & Ligand Pharmaceuticals & 12/9/1997 & 2/5/1999 \\
\hline Dexrazoxane & Zinecard & Pharmacia & $2 / 10 / 1992$ & $5 / 26 / 1995$ \\
\hline Docetaxel & Taxotere & Rhone-Poulenc Rorer & $7 / 27 / 1994$ & $5 / 14 / 1996$ \\
\hline Dolasetron mesylate & Anzemet & Hoechst Marion Roussel & 9/29/1995 & 9/11/1997 \\
\hline Dutasteride & Avodart & Glaxo Wellcome & $12 / 21 / 2000$ & $11 / 20 / 2001$ \\
\hline Epirubicin & Ellence & Pharmacia \& Upjohn & $12 / 15 / 1998$ & 9/15/1999 \\
\hline Erlotinib & Tarceva & Osi/Genentech & $7 / 30 / 2004$ & $11 / 18 / 2004$ \\
\hline Exemestane & Aromasin & Pharmacia \& Upjohn & 12/21/1998 & 10/21/1999 \\
\hline Finasteride & Proscar & Merck & 4/15/1991 & 6/19/1992 \\
\hline Fludarabine phosphate & Fludara & Berlex & 11/24/1989 & 4/18/1991 \\
\hline Fulvestrant & Faslodex & Astrazeneca & $3 / 28 / 2001$ & $4 / 25 / 2002$ \\
\hline Gefitinib & Iressa & Astrazeneca & $8 / 5 / 2002$ & $5 / 5 / 2003$ \\
\hline Gemcitabine hydrochloride & Gemzar & Lilly & 2/2/1995 & $5 / 15 / 1996$ \\
\hline Gemtuzumab ozogamicin & Mylotarg & Wyeth-Ayerst & 10/29/1999 & $5 / 17 / 2000$ \\
\hline Granisetron hydrochloride & Kytril & Smithkline Beecham & 4/14/1992 & 12/29/1993 \\
\hline Ibritumomab tiuxetan & Zevalin & Idec & $11 / 1 / 2000$ & 2/19/2002 \\
\hline Idarubicin hydrochloride & Idamycin & Adria Labs & 8/31/1989 & 9/27/1990 \\
\hline Imatinib mesylate & Gleevec & Novartis & $2 / 27 / 2001$ & $5 / 10 / 2001$ \\
\hline Irinotecan hydrochloride & Camptosar & Pharmacia \& Upjohn & 12/28/1995 & 6/14/1996 \\
\hline Lenalidomide & Revlimid & Celgene & $4 / 7 / 2005$ & $12 / 27 / 2005$ \\
\hline Letrozole & Femara & Novartis & $7 / 25 / 1996$ & $7 / 25 / 1997$ \\
\hline Levamisole hydrochloride & Ergamisol & Janssen & $11 / 1 / 1989$ & 6/18/1990 \\
\hline Masoprocol cream, 10\% & Actinex & Chemex/Reed \& Carnick & 4/10/1989 & 9/4/1992 \\
\hline Nelarabine & Arranon & Glaxosmithkline & $4 / 29 / 2005$ & $10 / 28 / 2005$ \\
\hline Nilutamide & Nilandron & Hoechst Marion Roussel & 3/7/1994 & 9/19/1996 \\
\hline Oxaliplatin & Eloxatin & Sanofi & $6 / 24 / 2002$ & $8 / 9 / 2002$ \\
\hline Paclitaxel & Taxol & Bristol-Myers Squibb & $7 / 22 / 1992$ & 12/29/1992 \\
\hline Palifermin (kgf) & Kepivance & Amgen & $6 / 24 / 2004$ & $12 / 15 / 2004$ \\
\hline Palonosetron & Aloxi & Helsinn Healthcare & $9 / 27 / 2002$ & $7 / 25 / 2003$ \\
\hline Pegaspargase & Oncospar & Enzon & $1 / 1 / 1991$ & 2/1/1994 \\
\hline Pemetrexed & Alimta & Eli Lilly & $9 / 30 / 2003$ & $2 / 4 / 2004$ \\
\hline Pentostatin & Nipent & Warner-Lambert & $2 / 11 / 1991$ & 10/11/1991 \\
\hline Porfimer & Photofrin & Qlt & $4 / 13 / 1994$ & 12/27/1995 \\
\hline Rasburicase & Elitek & Sanofi-Synthelabo & 12/16/1999 & $7 / 12 / 2002$ \\
\hline Rituximab & Rituxan & Genentech & 2/28/1997 & 11/26/1997 \\
\hline Samarium sm 153 lexidronam & Quadramet & Cytogen & 6/13/1995 & 3/28/1997 \\
\hline Sorafenib & Nexavar & Bayer/Onyx & $7 / 8 / 2005$ & $12 / 20 / 2005$ \\
\hline Temozolomide & Temodar & Schering-Plough & 8/13/1998 & 8/11/1999 \\
\hline Teniposide & Vumon & Bristol-Myers Squibb & 9/28/1990 & 7/14/1992 \\
\hline Topotecan hydrochloride & Hycamtin & Smithkline Beecham & 12/22/1995 & $5 / 28 / 1996$ \\
\hline Toremifene citrate & Fareston & Orion/Schering & 1/3/1995 & $5 / 29 / 1997$ \\
\hline Tositumomab-i131 & Bexxar & Corixa & $9 / 15 / 2000$ & $6 / 27 / 2003$ \\
\hline Trastuzumab & Herceptin & Genentech & $5 / 4 / 1998$ & 9/25/1998 \\
\hline Triptorelin pamoate & Trelstar Depot & Pharmacia & 6/26/1996 & $6 / 15 / 2000$ \\
\hline Valrubicin & Valstar & Anthra Pharmaceuticals & 12/31/1997 & 9/25/1998 \\
\hline Vinorelbine tartrate & Navelbine & Burroughs Wellcome & 8/27/1993 & $12 / 23 / 1994$ \\
\hline Zoledronic acid & Zometa & Novartis & 12/21/1999 & $8 / 20 / 2001$ \\
\hline
\end{tabular}

Abbreviation: NDA, new drug application. 


\begin{tabular}{|lcc|}
\hline \multirow{3}{*}{$\begin{array}{c}\text { Table 2. Regulatory Characteristics of New Therapeutic Oncology and } \\
\text { Other Drugs Approved in the United States, 1990-2005 }\end{array}$} \\
\hline & \multicolumn{3}{c}{$\%$} \\
\cline { 2 - 3 } Characteristic & Oncology Drugs & Other Drugs \\
\hline FDA priority rating* & 70.9 & 40.2 \\
Orphan drug designation & 48.5 & 18.5 \\
Expedited accesst & 47.1 & 13.4 \\
\hline *Therapeutic new molecular entities approved by FDA's Center for Drug \\
Evaluation and Research (CDER). \\
tDrugs that were developed under at least one of the following three FDA \\
regulatory mechanisms: subpart E, accelerated approval, fast track. \\
\hline
\end{tabular}

pharmaceutical sales in 2005 . We were able to identify 838 drugs that had entered the clinical testing pipeline for the first time anywhere in the world from 1993 to 2002. Of these drugs, 175 (21\%) were investigated for oncology indications. A somewhat higher proportion of the investigational oncology drugs are large molecules (28\%) than is the case for the approved drugs noted herein. The oncology drugs tended to be investigated for more indications than was the case for other investigational drugs. Whereas $46 \%$ of other investigational drugs were tested for more than one indication before an approval for marketing, $57 \%$ of the oncology drugs were investigated for multiple indications. More notably, nearly one third of the oncology drugs $(32 \%)$ were tested in at least four indications, whereas only $9 \%$ of the other drugs were examined in four or more indications before an original approval for marketing.

Figure 2 shows estimated clinical phase transition probabilities for investigational oncology drugs that first entered clinical testing from 1993 to 1997 and 1993 to 2002 . The results indicate that one half of the oncology drugs that entered the expensive phase III clinical testing phase never make it to US regulatory approval (although, the approval rate is somewhat higher when the longer timeframe for drugs entering clinical testing is considered). The product of the phase transition probability estimates yields an estimate of the clinical approval success rate for drugs entering the clinical testing pipeline. The results suggest that approximately one in five of the oncology drugs that entered the pipeline during 1993 to 1997 will eventually attain marketing approval, while the estimate improves to approximately one in four for the longer 1993 to 2002 period.

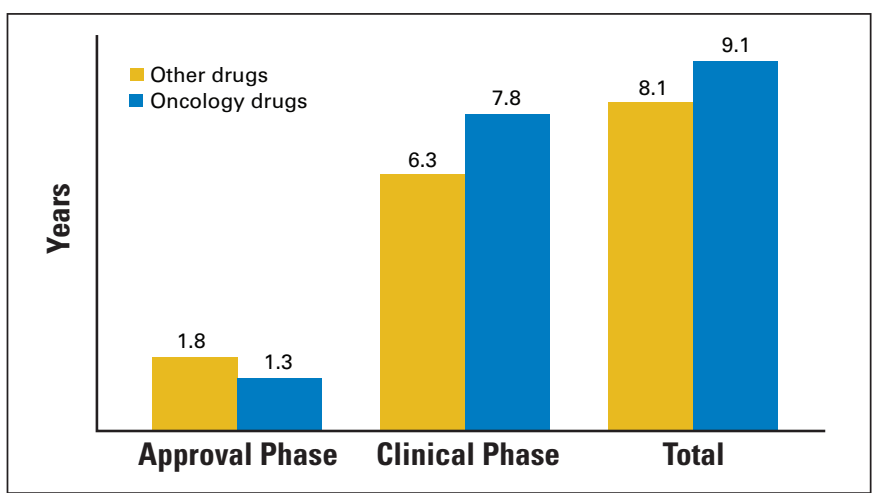

Fig 1. Mean clinical development and regulatory approval times for new oncology and other therapeutic molecular entities approved by the US Food and Drug Administration from 1990 to 2005.

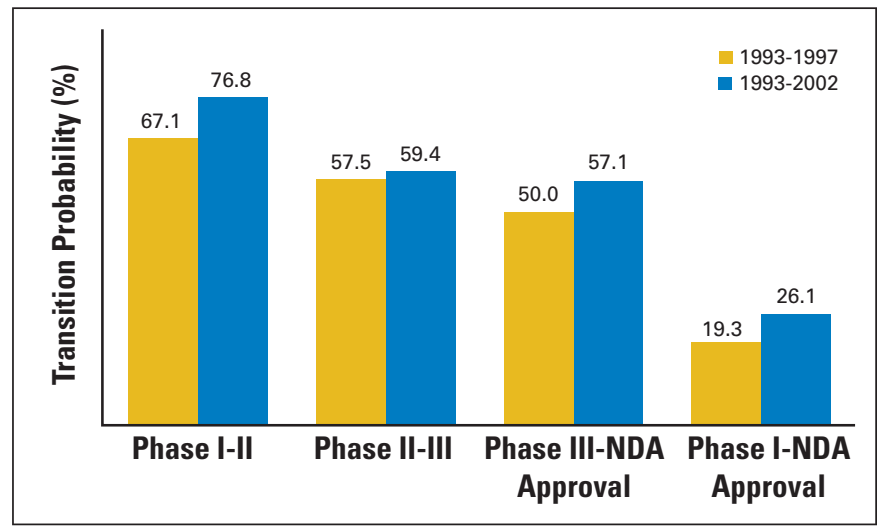

Fig 2. Clinical phase transition probabilities for investigational oncology compounds for the 20 largest firms by pharmaceutical sales (2005) by period during which compound first entered clinical testing. NDA, new drug application.

The results in Figure 2 are for all oncology drugs that were in the firms' clinical testing pipelines at some point. The data include compounds that were licensed in at some point in development by one of the firms and a smaller proportion of drugs that these firms licensed out to firms outside of the group of 20. Drugs that are licensed may have somewhat higher success rates than those that are developed entirely under the auspices of a given firm (self-originated) because of due diligence prescreening and because they tend to be licensed after the drugs had progressed to later clinical phases. Figure 3 shows estimates of phase transition probabilities and the overall clinical approval success rate for self-originated oncology drugs compared with the results for all oncology drugs. The self-originated compounds have a slightly lower approval success rate than is the case for all oncology drugs.

Finally, we examined transition probability and success rate results for oncology drugs compared with all other drugs. The results in Figure 4 cover all drugs for the entire 1993 to 2002 period. Oncology drugs have a higher likelihood of progressing to later clinical phases, but the success rate once drugs reach expensive phase III testing is notably lower for oncology drugs. Overall, though, the approval success rates for drugs entering the clinical testing pipeline are essentially the same.

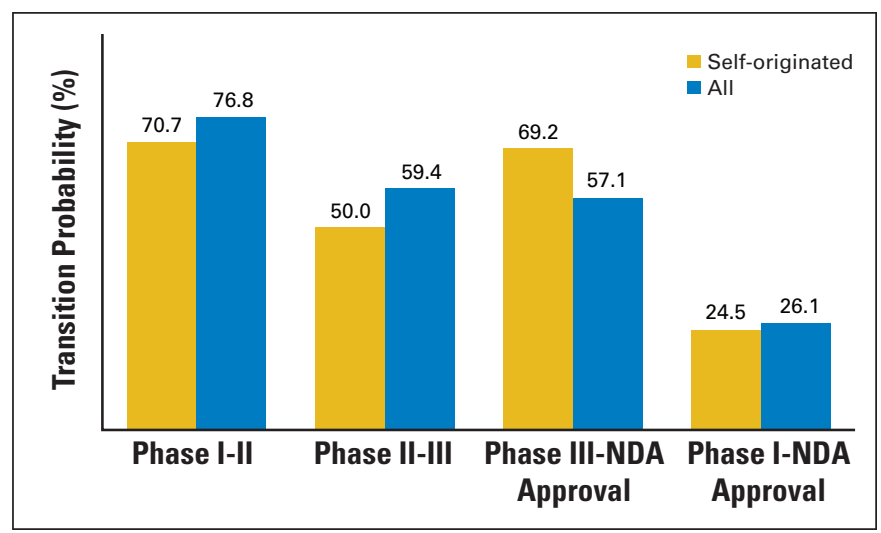

Fig 3. Clinical phase transition probabilities for investigational oncology compounds for the 20 largest firms by pharmaceutical sales (2005) by source of compound. NDA, new drug application. 


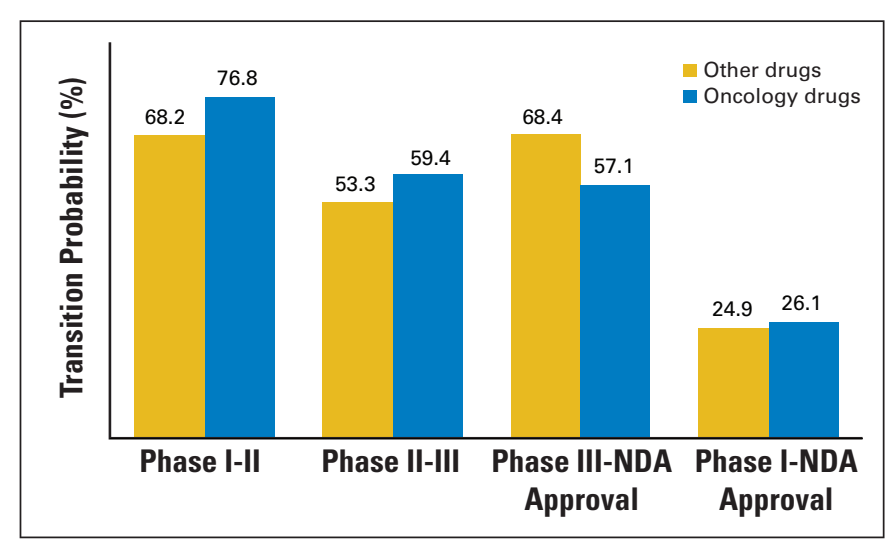

Fig 4. Clinical phase transition probabilities for investigational oncology and all compounds for the 20 largest firms by pharmaceutical sales (2005) for compounds that first entered clinical testing during 1993 to 2002. NDA, new drug application.

Biotech products, particularly mAbs, have become increasingly prevalent in oncology investigational drug pipelines. The data for the 20 firms examined here are too limited with regard to mAbs to provide reliable success rate estimates. However, for a recent analysis of biopharmaceutical R\&D costs, DiMasi and Grabowski ${ }^{13}$ examined clinical approval success rates for 522 recombinant proteins and mAbs that first entered clinical testing from 1990 to 2003 for what is likely either the population or something close to the population of such products. More than half (54\%) of the mAbs in this data set were examined for oncology indications. The clinical approval success rate for the biotech products in aggregate was $30 \%$, but only $19 \%$ for mAbs. Further analysis of that data set shows that the estimated success rate for the subset of oncology $\mathrm{mAbs}$ is also $19 \%$. The data do suggest, however, an increasing trend in success rates for mAbs in general.

\section{Market Attributes and Diffusion of Oncology Drugs: Comparative Trends}

In a recent article, Grabowski and Wang ${ }^{12}$ examine trends in various attributes of worldwide new drug introductions over the period 1982 to 2003. In particular, they consider trends in drug "innovativeness" as indicated by the number of first-in-class introductions. These are essentially new drugs with a novel mechanism of action. Second, they consider trends in the global diffusion of worldwide new drug introductions. In particular, they define a new drug as global when it is launched in a majority of the world's largest drug markets. Global diffusion is an indicator of both commercial as well as therapeutic importance. They also focus on the growth in biotech products and orphan drug products, two groups of products with increasing impact on the biopharmaceutical industry over the last two decades.

One key finding of the Grabowski and Wang ${ }^{12}$ analysis is that the number of first-in-class drug introductions has been increasing over time. This contrasts with a downward trend in overall new drug introductions that has been discussed by many observers. ${ }^{14}$ This latter trend has been cited as evidence for the declining research productivity of the pharmaceutical industry in recent years. However, this view must be qualified by the positive trend in drug innovativeness, as reflected by the increasing number of first-in-class products. Of course, therapeutic benefits are also obtained from follow-on introductions in a new drug class as well as by combination therapies involving new and established drugs. ${ }^{14}$ Significant drug progress occurs both by introduction of novel new classes and by the evolution of products in these classes after the first mover is introduced. ${ }^{15}$ However, first-in-class drug introductions represent important milestones in documenting the extent of drug innovation over time.

A second major finding of the Grabowski and Wang ${ }^{12}$ analysis is the increasing global character of new drug introductions. Grabowski and Wang found that nearly half (47\%) of all 1993 to 2003 new drug introductions were launched in a majority of the G7 countries. (The G7 countries were chosen as a relevant benchmark because they constitute the largest seven drug markets in terms of sales. These countries are the United States, Japan, the United Kingdom, Germany, France, Italy, and Canada.) This compares to 37\% for the 1982 to 1992 period. Furthermore, a prior study of new drug introductions for the 1970 to 1983 period found that only $24 \%$ of new drugs were characterized as global entities. ${ }^{16}$

Grabowski and Wang also found that biotech drugs account for a rising portion of all new drugs over the 1982 to 2003 period. The rapid growth of biotech compounds is reflected in the fact that biotech drugs accounted for only 4\% of worldwide introductions in the period 1982 to 1992 , but this increased to $16 \%$ in the 1993 to 2003 period. Furthermore, more than half of these biotech compounds originated in US firms. The growth of biotech drugs is particularly significant because they have been a major source of both first-in-class and global drugs. They also have a strong presence in the oncology class.

In this review article, we are particularly interested in how oncology drugs compare with other therapeutic classes with respect to these drug industry attributes considered in the Grabowski and Wang analysis. In this regard, Table 3 provides a breakdown of the distribution of new drugs by therapeutic areas and various subcategories using Grabowski and Wang's sample of 919 worldwide introductions for the 1992 to 2003 period. All therapeutic areas with 5\% or more of the total number of new drug introductions total are listed separately. The remaining areas with small numbers of introductions are combined into the miscellaneous category.

Table 3. Therapeutic Area Distribution of New Drugs for 1982-2003 Worldwide New Drug Introductions ${ }^{8}$

\begin{tabular}{|c|c|c|c|c|}
\hline Therapeutic Area & $\begin{array}{l}\text { All New } \\
\text { Drugs }\end{array}$ & $\begin{array}{c}\text { Global New } \\
\text { Drugs }\end{array}$ & $\begin{array}{l}\text { First-in- } \\
\text { Class New } \\
\text { Drugs }\end{array}$ & $\begin{array}{l}\text { Biotech } \\
\text { New Drugs }\end{array}$ \\
\hline Central nervous system & 130 & 57 & 12 & 1 \\
\hline Cardiovascular system & 128 & 45 & 7 & 5 \\
\hline Systemic anti-infectives & 127 & 62 & 12 & 6 \\
\hline Oncology & 99 & 52 & 21 & 25 \\
\hline Alimentary tract and metabolism & 86 & 29 & 13 & 9 \\
\hline Musculoskeletal system & 70 & 28 & 5 & 7 \\
\hline Blood and blood-forming organs & 59 & 24 & 9 & 15 \\
\hline Respiratory system & 57 & 21 & 5 & 2 \\
\hline Dermatologicals & 49 & 21 & 7 & 3 \\
\hline Miscellaneous & 118 & 49 & 24 & 18 \\
\hline \multicolumn{5}{|l|}{ Total } \\
\hline \multicolumn{5}{|c|}{$\begin{array}{l}\text { NOTE. Worldwide introductions by year are obtained from the IMS New } \\
\text { Product Focus database. A global new drug is defined as a new drug } \\
\text { introduced in a majority of the G7 countries. A first-in-class new drug is } \\
\text { defined as the first drug introduction in a specific five-digit Uniform System of } \\
\text { Classification category or a four-digit Anatomic Therapeutic Classification } \\
\text { category, based on information contained in the IMS databases. Biotech drug } \\
\text { classification is based on IMS designation in its New Product Focus database. } \\
\text { A few drugs are classified into more than one therapeutic area so category } \\
\text { totals may not equal the sum of the specific therapeutic areas. }\end{array}$} \\
\hline
\end{tabular}


Table 3 indicates that oncology was the fourth largest therapeutic area in terms of the number of worldwide introductions (99) behind the CNS, cardiovascular and systemic anti-infective categories. At the same time, the Table shows that oncology drugs had the most first-inclass and biotech drugs. It also ranked third in terms of global new drug introductions across all the therapeutic area categories. (The miscellaneous category is not included in this comparison, given that it's a conglomerate of many smaller drug categories.)

It is instructive to consider the share of oncology drugs that embody these various attributes compared with other major drug classes. Consider this information for the four largest therapeutic areas in Table 3: the CNS, cardiovascular, anti-infective, and oncology categories. Oncology is particularly distinguished by the large percentage of its new drug introductions that were first-in-class. Over the 1992 to 2003 period, $21 \%$ of oncology introductions were first-in-class entities, as compared with less than $10 \%$ of the introductions in the other three classes. This figure also shows that more than half of all oncology introductions were classified as global drugs as compared with 35\% to $49 \%$ of the drugs in the other three therapeutic areas.

The results in Table 3 demonstrate that the oncology therapeutic area has been a focal point for the introduction of innovative first-inclass compounds with a high rate of global diffusion. Oncology also has been an increasing focus of biotech drug R\&D. As can be seen from the data in Table 3, 25\% of oncology drug approvals are based on biotechnology techniques, compared with $5 \%$ or less in the three other major classes. This is a striking difference. Biotech products are an important driver of strong innovative performance observed for the oncology class in recent years.

\section{Orphan Drug Act and Oncology Drugs}

The oncology drug class has benefited from the passage of the Orphan Drug Act in 1983. The Act specifically applies to illness or conditions with a prevalence of less than 200,000 individuals. This Act created a number of incentives designed to spur R\&D investment for rare conditions and illnesses. ${ }^{17,18}$ First, the Act instructed the FDA to implement new protocols to facilitate orphan drug approvals, or advanced counseling to create a more effective R\&D process. Second, Congress created a 50\% tax credit for clinical trial expenditures for orphan drug designations. Third, a 7-year marketing exclusivity was granted for FDA-designated orphan drug indications, apart from any patent protection that existed on these drugs.

These provisions have been an important catalyst for the development of oncology drugs for rarer forms of cancer. As noted herein, nearly half of the oncology drugs introduced had an orphan indication approved at the time of initial marketing approval. Once again, this is a much higher percentage than what is observed for other drugs. In an earlier analysis ${ }^{17}$ of the first dozen years of the US Orphan Drug Act, the authors found that a total of 502 approved drugs and clinical drug candidates obtained orphan drug designation from the FDA. The leading indication category was cancer with 89 drug entities $(17.7 \%$ of all drugs that received an orphan drug designation).

Grabowski ${ }^{18}$ has analyzed the distribution of sales for 27 orphan drugs introduced in the 1990 to 1994 period. While there are a few orphan products with large annual sales, the median orphan drug in this sample had peak annual worldwide sales of only \$29.5 million. The median nonorphan introduction over this same period had peak global sales of \$236 million. ${ }^{6}$
The group of 27 orphan compounds introduced from 1990 to 1994 included six cancer treatments (the largest indication category). All of these six drugs also received a priority drug rating from the FDA as well as orphan drug designation. (The six cancer drug introductions receiving orphan drug approval over the 1990-1994 period were altretamine, cladribine, fludarabine phosphate, idarubicin, pentostatin, and teniposide.) These six orphan therapies had peak global sales that ranged from \$2 million to \$103 million. The median and mean global peak sales for this set of orphan cancer treatments were $\$ 12$ million and $\$ 27$ million, respectively.

The Orphan Drug Act clearly has been an important stimulant of new cancer treatments for small patient populations with correspondingly modest levels of sales. Orphan drugs can realize a positive return on investment with smaller sales levels than can non-orphan products given their very different economics. First, as discussed, they have much smaller up-front R\&D costs, with typically smaller sized clinical trials and the special tax credits. In addition, orphan drugs often have fewer competitors and are typically prescribed primarily by specialists. Hence, they tend to have lower promotional and distribution costs.

\section{Commercial Significance of Oncology Drugs}

As in the case of other therapeutic categories, the distribution of sales for cancer drugs is highly skewed. Although the oncology class includes a number of orphan drug compounds, it also has many drugs that are in the top ranks of all drug products. These are products with annual worldwide sales in excess of $\$ 1$ billion. With an increased knowledge of the molecular basis of cancer, the oncology class has been characterized in recent years by the introduction of therapeutically important monoclonal antibodies and other targeted pharmaceutical agents. These include rituximab, trastuzumab, imatinib, and bevacuzimab. All of these drugs have reached the market between 1997 and 2004. These are now among the leading drug therapies ranked in terms of sales.

The oncology drug class is the fastest growing therapeutic category of all the major drug classes in terms of market sales. The rapid growth of the cancer area is reflected in the annual sales of the top 200 pharmaceuticals. Med Ad News chronicles this each year. Our analyses of development candidates and marketed products abstracts from the supportive care products used to ameliorate adverse effects such as anemia, neutropenia, and nausea and vomiting that are frequently experienced with treatments of diseases such as cancer and AIDS. It is worth noting that the leading biologically derived drugs for anemia and neutropenia have also experienced rapid growth in recent years. For example the supportive care products for the two conditions (for example, Procrit/Eprex [epoetin alfa; Amgen, Thousand Oaks, CA], Aranesp [darbepoetin alfa; Amgen], Epogen [epoetin alfa; Amgen], and Neulasta/Neupogen [Pegfilgrastim/Filgrastim; Amgen] had global sales of $\$ 14.3$ billion in 2005 compared with $\$ 7.6$ billion in 2001), according to the MedAd News' Surveys of 200 BestSelling Prescription Drugs for these years. ${ }^{19}$ The Med Ad list of the top 200 selling pharmaceuticals for 2001 included 14 cancer drugs with a total of $\$ 10.5$ billion dollars worldwide sales. ${ }^{19}$ There was only one single drug entity (Taxol; paclitaxel; BristolMyers Squibb, New York, NY) that had sales in excess of \$1 billion. By contrast, the 2005 list of top 200 pharmaceuticals had $\$ 23.5$ billion in total cancer drug sales with 11 of 18 cancer drugs on the list with sales in excess of $\$ 1$ billion. ${ }^{20}$ 
The rapid growth of sales in the cancer area has been driven by the fast uptake of targeted breakthrough drug products such as Rituxan (rituximab; Genentech, South San Francisco, CA), Gleevec (imatinib mesylate; Novartis, East Hanover, NJ), Herceptin (trastuzumab; Genentech), and Avastin (bevacizumab; Genentech). These four products by themselves accounted for $\$ 8.4$ billion in worldwide sales in 2005 and were the four largest selling cancer treatments in the first quarter of 2006. ${ }^{21}$ There is also evidence of a strong pipeline of targeted therapies in development in both the biotechnology and pharmaceutical industry. This is being fostered by the increasing knowledge base emanating from basic biomedical research as well as a favorable economic environment for drug innovations in the oncology therapeutic area. ${ }^{21}$ Hence, the continued introduction of innovative new oncology products is likely to occur over the foreseeable future.

\section{CONCLUSION}

In comparison with other new drugs, new oncology drugs tend to be distinctly different in terms of regulatory status and development metrics. A substantial majority of first approvals for marketing of oncology drugs (71\%) have received priority reviews of their marketing applications from the FDA for drugs approved in the United States from 1990 to 2005 . This compares with the $40 \%$ rate at which other new drugs received a priority review status from the FDA. Approved oncology drugs have also had a disproportionately higher share of orphan drug approvals, and approved oncology drugs were able to take advantage of FDA programs to speed development at a rate that was 3.5 times higher than that for other new drugs.

Despite more often obtaining regulatory advantages from programs to speed clinical development and regulatory review of marketing applications, US clinical development times and total times from the start of US clinical testing to marketing approval were longer, on average, for oncology drugs than for other drugs approved from 1990 to 2005. The recent efforts by the FDA to establish new approaches to assess the efficacy and safety of investigational drugs through its Critical Path Initiative hold the promise of shorter development times for oncology and other drugs. ${ }^{22}$ Of particular relevance for oncology drugs is the Critical Path Initiative's goal to find and validate new biomarkers.

Although we found that oncology and other drugs had marketing approval success rates for drugs entering the clinical testing pipeline that were similar, a higher percentage of oncology drugs failed after entering phase III testing than did other drugs. Phase III is generally the most expensive clinical development phase. If all other things were equal, this result would imply higher average development costs for oncology drugs (taking into account the costs of drug failures). Methods to better prioritize the choice of investigational oncology drugs for a transition from phase II to phase III testing could yield substantial gains. As has been suggested elsewhere, a greater investment in gathering appropriate information in phase I and II trials can help achieve this objective. ${ }^{23}$
The full capitalized cost per approved new drug (cash flow plus time costs) depends on out-of-pocket expenditures, approval success and phase attrition rates, and development and regulatory approval times. ${ }^{5}$ On the basis of data obtained from a commercial business intelligence database, Adams and Brantner ${ }^{24}$ estimated the R\&D cost per approved new drug for development that occurred primarily during the 1990 s to be $20 \%$ higher for oncology drugs compared to the average for all drugs ( $\$ 1,042$ million compared to $\$ 868$ million), although they also provide an estimate for breast cancer development that is $30 \%$ below average. However, their cost differentials by therapeutic class were based solely on differences in estimated clinical approval and phase transition rates, and development times. (Through a number of simulation experiments, DiMasi ${ }^{25}$ demonstrates the extent to which $R \& D$ cost estimates are sensitive to changes in clinical approval and phase transition rates, and development times.) They did not have data on differences in cash flows by class. To our knowledge, there have been no published studies with enough data on oncology drug R\&D cash flows to provide estimates of average oncology out-of-pocket R\&D costs. A number of factors determine out-of-pocket expenditures, including discovery costs; R\&D costs for chemistry, manufacturing, and controls; the costs of providing clinical testing supplies; infrastructure costs; the complexity of treating patients with the conditions investigated; the number of subjects tested per indication; and the number of indications investigated before approval. We currently know very little about most of these factors, although results presented here suggest that oncology drugs tend to be tested in many more uses before first marketing approval than is the case for drugs in general.

Despite the considerable hurdles in developing new oncology drugs, the new oncology drugs that have been approved in recent years have been relatively novel and commercially successful. The evidence indicates that approved oncology drugs were more often first-in-class and diffused more widely to major international markets than was the case for drugs in other classes. Nonetheless, the need for new therapies that are more effective and safe is still substantial. The incentives and scientific opportunities to develop highly effective and safer oncology drugs in the future will depend critically on public-sector investment in basic research, developments in translational medicine, and regulatory reforms that advance drug-development science. ${ }^{22}$

\section{AUTHORS' DISCLOSURES OF POTENTIAL CONFLICTS} OF INTEREST

The authors indicated no potential conflicts of interest.

\section{AUTHOR CONTRIBUTIONS}

Conception and design: Joseph A. DiMasi, Henry G. Grabowski Collection and assembly of data: Joseph A. DiMasi, Henry G. Grabowski

Data analysis and interpretation: Joseph A. DiMasi, Henry G. Grabowski

Manuscript writing: Joseph A. DiMasi, Henry G. Grabowski Final approval of manuscript: Joseph A. DiMasi 


\section{REFERENGES}

1. Miller $S$, Parker $A$, Peterson $C$, et al: Drug Trend Report 2005. St Louis, MO, Express Scripts, 2006

2. Ricks $D$ : Experts question costly cancer care Newsday, June 3, 2006, p A8

3. Mitchell S: Analysis: Cancer drug pipeline on the rise. United Press International, July 12, 2006, http://www.upi.com/HealthBusiness/view.php?StorylD $=20060818-034618-2296 \mathrm{r}$

4. Pollack A: New drugs for cancer could soon flood the market. New York Times, June 5, 2006, p C2

5. DiMasi JA, Hansen RW, Grabowski HG: The price of innovation: New estimates of drug development costs. J Health Econ 22:141-185, 2003

6. DiMasi JA, Grabowski HG, Vernon J: R\&D costs and returns by therapeutic category. Drug Inf J 38:211-223, 2004

7. DiMasi JA, Hansen RW, Grabowski HG, et al: Cost of innovation in the pharmaceutical industry. $J$ Health Econ 10:107-142, 1991

8. DiMasi JA: Success rates for new drugs entering clinical testing in the United States. Clin Pharmacol Ther 58:1-14, 1995

9. Gosse ME, DiMasi JA, Nelson TF: Recombinant protein and therapeutic monoclonal antibody drug development in the United States: 1980-1994. Clin Pharmacol Ther 60:608-618, 1996

10. DiMasi JA: Risks in new drug development: Approval success rates for investigational drugs Clin Pharmacol Ther 69:297-307, 2001

11. Abtantes-Metz RM, Adams CP, Metz AD: Pharmaceutical development phases: A duration analysis. J Pharm Fin Econ Policy 14:19-41, 2005

12. Grabowski HG, Wang YR: The quantity and quality of worldwide new drug introductions 1982 2003. Health Aff (Millwood) 25:452-460, 2006

13. DiMasi JD, Grabowski HG: The cost of biopharmaceutical R\&D: Is biotech different? Manag Dec Econ (in press)

14. Berndt ER, Cockburn IM, Grepin K: The impact of incremental innovation in pharmaceuticals: Drug utilization in original and supplemental indications. Pharmacoeconomics 24:69-86, 2006 (suppl 2 )

15. DiMasi JA, Paquette C: The economics of follow-on drug R\&D: Trends in entry rates and the timing of development. Pharmacoeconomics 20:11 29, 2004 (suppl 3)

16. Grabowski HG: An analysis of US international competitiveness in pharmaceuticals. Manag Dec Econ, Special Issue: 27-33, 1989

17. Shulman SR, Manocchia M: The US Orphan Drug programme: 1983-1995. Pharmacoeconomics 12:312-326, 1997
18. Grabowski HG: 2005: Increasing R\&D incentives for neglected diseases: Lessons from the Orphan Drug Act, in Maskus KE, Reichman JH (eds): International Public Goods, and Transfer of Technology Under a Globalized Intellectual Property Regime. Cambridge, United Kingdom, Cambridge University Press, 2005, pp 457-480

19. Med Ad News 200 - World's Best Selling Medicines - Sales. Med Ad News 21, 2002

20. Med Ad News 200 - World's Best Selling Medicines - Sales. Med Ad News 25:22-28, 2006

21. Bank of America, Equity Research, Health Care Group: Cancer Remains Large Opportunity. NY, Bank of America, 2006, pp 18-31

22. Food and Drug Administration: Challenge and Opportunity on the Critical Path to New Medical Products. Washington, DC, US Department of Health and Human Services, 2004. http://www.fda gov/oc/initiatives/criticalpath/whitepaper.pdf

23. Roberts TG, Lynch TJ, Chabner BA: The phase III trial in the era of targeted therapy: Unraveling the "go or no go" decision. J Clin Onco 21:3683-3695, 2003

24. Adams $C P$, Brantner $\mathrm{V}$ : Estimating the cost of new drug development: Is it really $\$ 802$ million? Health Aff 25:420-428, 2006

25. DiMasi JA: The value of improving the productivity of the drug development process: Faster times and better decisions. Pharmacoeconomics 20:1-10, 2002 (suppl 3) 